\title{
MÉTODO HÍBRIDO PARA DETECÇÃO E REMOÇÃO DE SOMBRAS EM IMAGENS
}

\author{
Dr. Marcos Batista Figueredo ${ }^{1}$; Eugenio Rocha Silva Junior ${ }^{2}$ \\ ${ }^{1}$ Universidade do Estado da Bahia - UNEB, Departamento de Ciências Exatas e da \\ Terra - DCET II; Alagoinhas/Bahia; mbfigueredo@uneb.br \\ ${ }^{2}$ Universidade do Estado da Bahia - UNEB, Departamento de Ciências Exatas e da \\ Terra - DCET II; Alagoinhas/Bahia; eugeniojunio99@gmail.com
}

Resumo: Sombras em uma imagem podem distorcer informações sobre a forma e orientação do objeto e até sobre a fonte de luz. Assim, a detecção e remoção de sombras é uma tarefa crucial e inevitável para alguns processos de visão computacional. Este artigo propõe uma estrutura simples usando o espaço de cores HSV para detectar e remover sombras de imagens. Inicialmente, uma abordagem probabilística da intensidade no espaço de cores HSV é proposta para a detecção de sombras. Depois que as sombras são identificadas, um modelo de densidade de sombra é aplicado A principal contribuição do modelo proposto é que, após a remoção de sombras, não há transição brusca entre as partes sombreadas e as partes não sombreadas, e todos os detalhes nas regiões sombreadas permanecem intactos.

Palavras-Chave: Detecção de sombras; Remoção de Sombras; HSV; Visão Computacional.

\section{HYBRID METHOD FOR DETECTION AND REMOVAL OF IMAGE SHADOWS}

Abstract: Shadows in an image can distort information about the shape and orientation of the object and even about the light source. Thus, detecting and removing shadows is a crucial and inevitable task for some computer vision processes. This article proposes a simple structure using HSV color space to detect and remove shadows from images. Initially, a probabilistic approach to HSV color space intensity is proposed for shadow detection. Once shadows are identified, a shadow density model is applied. The main contribution of the proposed model is that after the removal of shadows, there is no sharp transition between the shaded parts and the unshaded parts, and all the details in the regions. Shaded shades remain intact.

Keywords: Shadow detection; Shadow Removal; HSV; Computer vision. 


\section{INTRODUÇÃO}

A remoção de sombras é um problema fundamental e desafiador no campo de processamento de imagens. Uma sombra pode ser caracterizada como uma obstrução da luz que incide sobre um objeto e as cria. As áreas de sombra são menos iluminadas do que as áreas circundantes e em alguns casos, as sombras fornecem informações úteis, como a posição relativa de um objeto da origem, mas em geral, eles causam problemas em diversas aplicações de visão computacional, como segmentação, detecção e contagem de objetos.

Com base na intensidade, [1] propõe que as sombras sejam de dois tipos: intensas e simples. As sombras simples retêm a textura da superfície de fundo, enquanto as sombras intensas são muito escuras e têm pouca preservação da textura. Assim, a detecção de sombras é complicada, pois podem ser confundidas com objetos escuros em vez de sombras. Embora a maioria dos métodos de detecção de sombras precise de várias imagens para a calibração da câmera, a melhor técnica deve ser capaz de extrair sombras de uma única imagem. Este artigo propõe uma estrutura simples usando o espaço de cores formadas pelas componentes Hue (matiz), Saturation (saturação) e Value (valor) a (HSV) para detectar e remover sombras de imagens sombreadas.

Neste contexto, um método simples é inicialmente proposto, o qual requer informações de intensidade no espaço de cores HSV para detecção de sombras. Então a imagem é segmentada de acordo com a densidade da sombra. Finalmente, as sombras são removidas reestruturando cada pixel no espaço de cores HSV. Além disso, as cores da região recuperada são corrigidas no espaço de cores vermelhoverde-azul (RGB).

É mais fácil para o olho humano distinguir sombras de objetos. No entanto, a identificação de sombras por computador é um problema de pesquisa desafiador. É, portanto, de grande importância descobrir maneiras de detectar corretamente as sombras e removê-las, mantendo intactos os outros detalhes da imagem original. Uma quantidade significativa de pesquisa foi realizada na detecção e remoção de sombras nos últimos anos.

A importância de poder separar estes efeitos na imagem tem sido bem estudada na literatura [2-4]. Ao ponto de podermos separar esta atividade em duas categorias: métodos operando no domínio do gradiente e que operam no domínio da intensidade.

A primeira abordagem remove as sombras com base na manipulação do domínio do gradiente, [4-6], que opera sobre as diferenças entre os pixels vizinhos, e não sobre os valores de pixel diretamente. Uma ideia comum nessa técnica é anular o gradiente nos limites de sombra e reconstruir o resultado sem sombras, utilizando as informações obtidas da região.

O segundo depende da precisão detecção das bordas sombreadas e pode não 


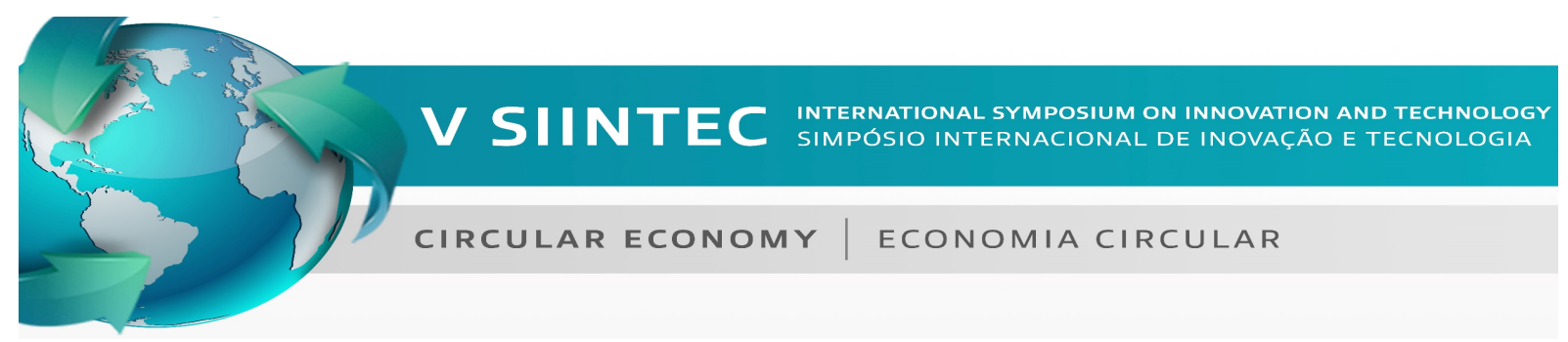

produzir resultados devido à esta imprecisão. Difundido por [7] este procedimento requer muita interação do usuário para especificar a região sombreada. A técnica aplica uma equação de Poisson [8], que construiu um sistema livre de sombras para a área especificada consistente com a textura relativa entre a sombra e a região exterior a seu contorno.

O trabalho de [9] utiliza um classificador que prevê um rótulo para cada região. Com isso esperou-se minimizar o erro associado a interação discutida por [7]. Na mesma linha [10] apresenta um esquema de reconhecimento e remoção de sombra baseado em aprendizagem. No trabalho de [11] é apresentado uma modelagem da superfície de intensidade 3D. Nesta, a superfície com intensidade adequada de iluminação, pode ser obtida com base na correspondente à mesma textura de não-sombra. Já [12] propõe uma adaptação de métodos que decompõe a imagem de entrada em manchas sobrepostas de acordo com a distribuição de sombras.

A métodos dos trabalhos discutidos necessitam de várias imagens, câmeras calibrada específicas, interação do usuário com a sombra ou ainda métodos como a reintegração usando uma equação de Poisson que consomem tempo de processamento. Além disso, objetos escuros são frequentemente confundidos como sombras.

\section{MÉTODO}

\subsection{Detecção da Sombra}

O modelo HSI (que pode ser encontrado como HSV) é um modelo que se aproxima mais da maneira como enxergamos a cor. Da mesma maneira que o RGB, uma cor no modelo HSI pode ser representada por uma tripla: $f(x, y)=(H, S, I)$ em que $H$ é o tom ou Matiz (hue), S é a saturação e I a intensidade. Ja se observou[11] que existe uma relação entre a matiz e a intensidade em relação a sobras o que permite detectar sombras de imagens coloridas. Foi observado que as regiões de sombra geralmente têm um valor de intensidade menor e um valor de matiz mais alto em comparação com outras regiões não sombreadas na imagem. Essa proporção é dada pela equação (1):

$$
r(x)=H(x) /(I(x)+0.01)
$$

onde $I(x)$ e $H(x)$ referem-se ao valor de intensidade e matiz do pixel $x$ na imagem de entrada, respectivamente. Aplicamos o filtro bilateral para aliviar o efeito de ruído antes de calcular o mapa de razão $r(x)$. É importante destacar que deixamos $r(x)$ entre $[0 ; 255]$ para torná-lo mais conveniente para o processamento subsequente.

Ao obter o mapa $r(x)$, usamos o método de Otsu [15] para destacar a região sombreada da imagem ainda de forma grosseira. A escolha do método de Otsu foi determinada pela resposta diante aos demais já descritos na literatura. Ele, determina um valor ideal para um "threshold" que separa os elementos do fundo e da frente da imagem em dois clusters, atribuindo a cor branca ou preta para cada um deles. $O$ Aplicamos sobre o histograma do mapa $r(x)$ para selecionar automaticamente um limiar ótimo $T$, que é determinado da seguinte forma: Supondo que a intensidade de 
cinza na imagem é dado por intervalo $[1,2, \ldots, L]$ sendo cada entrada definida por $x_{i}$ com $1 \leq \mathrm{i} \leq \mathrm{L}$. A distribuição de probabilidade para estes níveis de cinza são determinados pela equação 2 :

$$
p(i)=x_{i} / X
$$

Com $x_{i} \geq 0$ e $X=\sum_{i=1}^{L} x_{i}$. A imagem é divida em duas partes $\mathrm{C}_{0}$ e $\mathrm{C}_{1}$ as quais chamamos de frente e fundo separados pelo limiar $t$. Temos que os pixels de $\mathrm{C}_{0}$ são representados no níveis $[1,2, \ldots, t]$ e $C_{1}$ variando de $[t+1, \ldots, L]$. Assim nosso $T$ ótimo será dado pela equação:

$$
\mathrm{T}=\operatorname{argmax}\left\{\omega_{0}\left(\mu_{\mathrm{o}}-\mu_{\mathrm{T}}\right)^{2}+\omega_{1}\left(\mu_{1}-\mu_{\mathrm{T}}\right)^{2}\right\}
$$

Em que: $\mu_{T}=\sum_{i=1}^{L} i . p(i), \mu_{1}=\sum_{i=t+1}^{L} i . p(i) / \omega_{1}, \mu_{0}=\sum_{i=1}^{L} i . p(i) / \omega_{0}, \omega_{1}=\sum_{i=t+1}^{L} p(i)$, $\omega_{0}=\sum_{i=1}^{L} p(i)$. De acordo com o limiar T, um mapa, grosseiro, da região de sombra $s$ pode ser obtido pela equação 4 :

$$
s(x)=\left\{\begin{array}{c}
1, r(x)>T \\
0, c c
\end{array}\right.
$$

onde $s(x)$ se refere aqueles pixels localizados nas regiões com maior probabilidade de existir uma sombra. Aqui usamos uma abordagem pouco diferente da literatura ao aplicarmos um esquema de limiar sucessivo, que é apresentado [4]. Apesar de apresentar diversos parâmetros de limiarização a escolha se deu por experimentação.

\subsection{Remoção da Sombra}

Para remoção de sombra, um modelo simples de sombra é usado, onde existem dois tipos de fonte de luz: direta e luz ambiente. A luz direta vem diretamente da fonte, enquanto a luz do ambiente é proveniente de reflexos das superfícies circundantes. $O$ modelo de sombra pode ser representado pela seguinte fórmula (5):

$$
I_{l}=\left(t_{i} \cos \theta_{t} L_{d}+L_{e}\right) R_{i}
$$

onde $l_{1}$ representa o valor para o i-ésimo pixel, $L_{d}$ e $L_{e}$ representam as intensidade dos pixels que caracterizam não-sombra e dos pixels da sombra, $R_{i}$ é a refletância da superfície desse pixel, e $\theta_{\mathrm{t}}$ é o ângulo entre a direção direta da iluminação e a superfície normal. $t_{i}$ é o fator de atenuação da luz direta; se $t_{i}=1$, o ponto de objeto está em uma região de luz do sol e, se ti $=0$, o ponto de objeto está em uma região de sombra. Aqui, um coeficiente de sombra para o i-ésimo pixel é denotado por $\mathrm{k}_{\mathrm{i}}=$ $t_{i} \cos \theta_{l}$ e a razão entre pixels não-sombra e pixels de sombra pode ser calculada como $r=L_{d} / L_{e}$.

O procedimento de detecção de sombras nos fornece uma máscara de sombra binária onde cada pixel i recebe um valor $k_{i}$ de 1 ou 0 . Com base nesse modelo, o objetivo é 


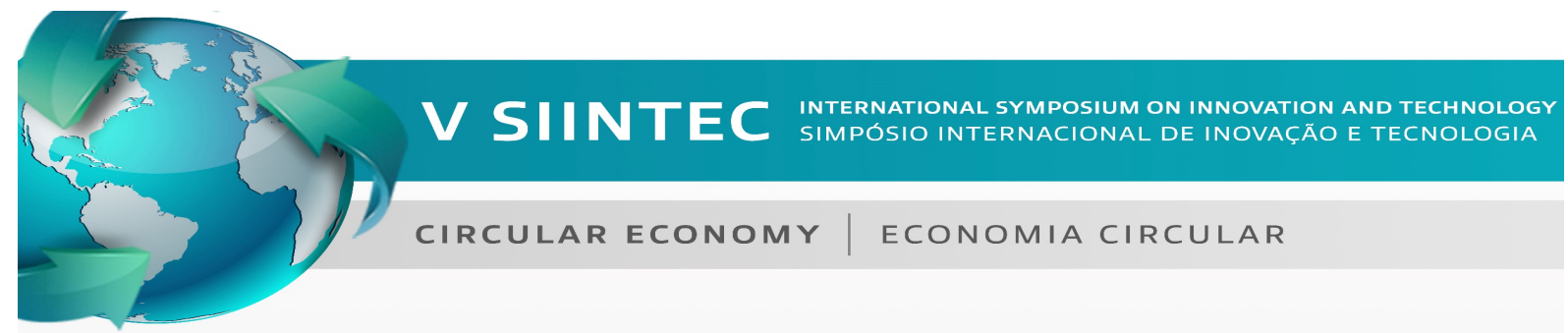

relimar cada pixel usando esse coeficiente para obter uma imagem livre de sombras. O novo valor de pixel é calculado com base na seguinte fórmula:

$$
I_{i}^{\text {Livre de Sombra }}=\left((r+1) /\left(k_{i} r+1\right)\right) I_{i}
$$

Inicialmente, as intensidades médias de pixel nas áreas de sombra e não-sombra da imagem são calculadas, e essa diferença é adicionada aos pixels do canal I. Em seguida, calcula-se a relação entre os pixels de sombra médios e os pixels médios não sombreados. Em seguida, os valores de H e S são calculados. Depois disso, a imagem é convertida em uma imagem RGB. Por causa da luz ambiente, as proporções dos dois pixels não são iguais em todos os três canais de cores. Os dois cantos serão diferenciados apenas na intensidade, mas também na maturação e saturação. Assim, corrigir a intensidade dos pixels sombreados não remove a sombra e também precisamos corrigir os valores de cromaticidade.

Apos uma filtragem, a densidade de sombra é calculada, o que mostra o grau do efeito da luz. Se torna 0 em uma região de sol, e se torna 1 em uma região de penumbra. Usando a densidade da sombra, a área de sombra é segmentada nas regiões da luz do sol e de penumbra. Como a cor da luz da região da penumbra nem sempre é a mesma da região do sol, o ajuste de cor é realizado entre elas. Em seguida, a média de cor e a variância da região da penumbra são ajustadas para serem as mesmas da região ensolarada. Nos ajustes de penumbra, cor e brilho para pequenas regiões são realizados da mesma forma que são para a região da penumbra. Finalmente, todas as fronteiras entre regiões sombreadas e regiões vizinhas iluminadas são suavizadas, convolvendo-as com uma máscara gaussiana.

\section{RESULTADOS}

O módulo de detecção e remoção de sombras foi implementado no ambiente MATLAB. Numa máquina Intel Pentium i5 $2.40 \mathrm{GHz}$ com um sistema operacional de 64 bits e 2 GB de RAM foi usada para testes. Nos experimentos, foram utilizadas imagens de tamanho 256X256. O conjunto de treinamento consistiu em 40 imagens ao ar livre e 40 imagens internas. Alguns exemplos de detecção de sombras e remoção de sombras sob a estrutura proposta são mostrados na Figura 1 e 2.

As duas primeiras linhas da Figura 1 mostram resultados de detecção de sombra de imagens externa. A Figura 2 mostra uma imagem livre de sombra usando a estrutura de remoção de sombra proposta. Vemos que o framework proposto pode remover com sucesso as regiões sombreadas porem com uma certa discrepância de coloração.

A tabela 1 apresenta uma comparação entre a estrutura proposta e alguns métodos bem relatados na literatura. Nela, vemos que nossa abordagem supera os métodos propostos nas taxas de detecção e remoção. O tempo médio de cálculo para a detecção de sombras e remoção do modelo é mostrado na Tabela 2. 


\section{\begin{tabular}{l|l} 
CIRCULAR ECONOMY & ECONOMIA CIRCULAR
\end{tabular}}

Figura 1: Modelo de detecção de sombra localizando sombra em imagens externas
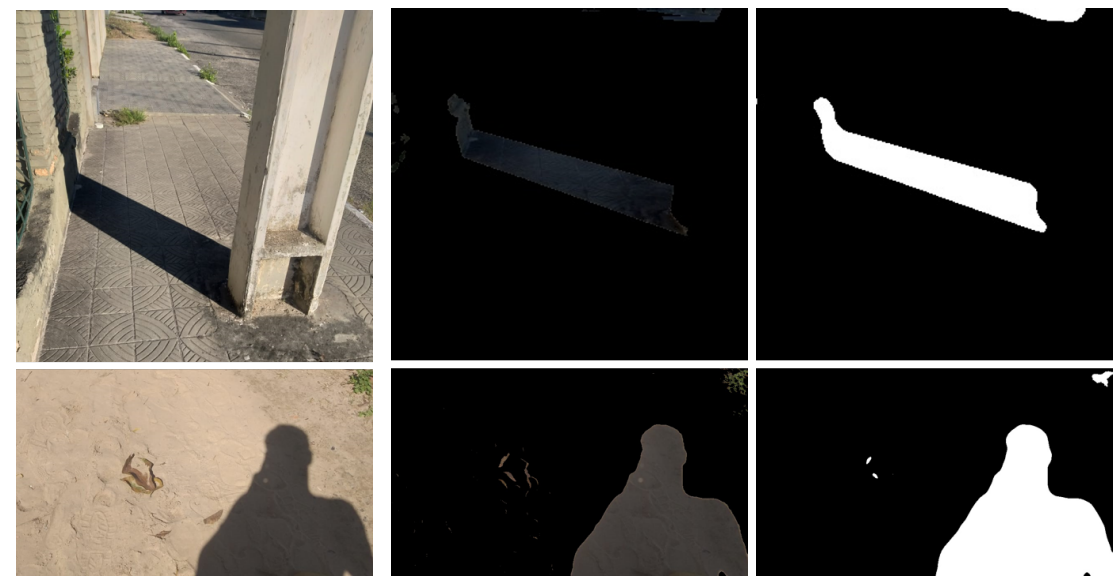

Figura 2: Remoção da sombra

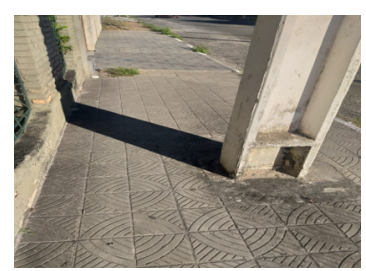

(a) Imagem Original

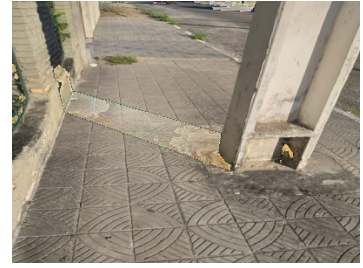

(b) Remoção da sombra

Tabela 1. Comparação entre taxa de detecção e remoção

\begin{tabular}{|c|c|c|}
\hline Modelo & Detecção Sombra & Remoção Sombra \\
\hline Fei Kou [4] & $86,21 \%$ & $82,45 \%$ \\
\hline Anat Levin [5] & $82,53 \%$ & $81.22 \%$ \\
\hline G D Finlayson [7] & $93,54 \%$ & $94,32 \%$ \\
\hline Método Proposto & $95,32 \%$ & $95,34 \%$ \\
\hline
\end{tabular}

Tabela 2. Comparação entre tempo médio de detecção

\begin{tabular}{|l|c|c|}
\hline \multicolumn{1}{|c|}{ Modelo } & Detecção Sombra (s) & Remoção Sombra (s) \\
\hline Fei Kou [4] & 0.880 & 0.9236 \\
\hline Anat Levin [5] & 0.0674 & 0.8209 \\
\hline G D Finlayson [7] & 0.0543 & 0.8198 \\
\hline
\end{tabular}




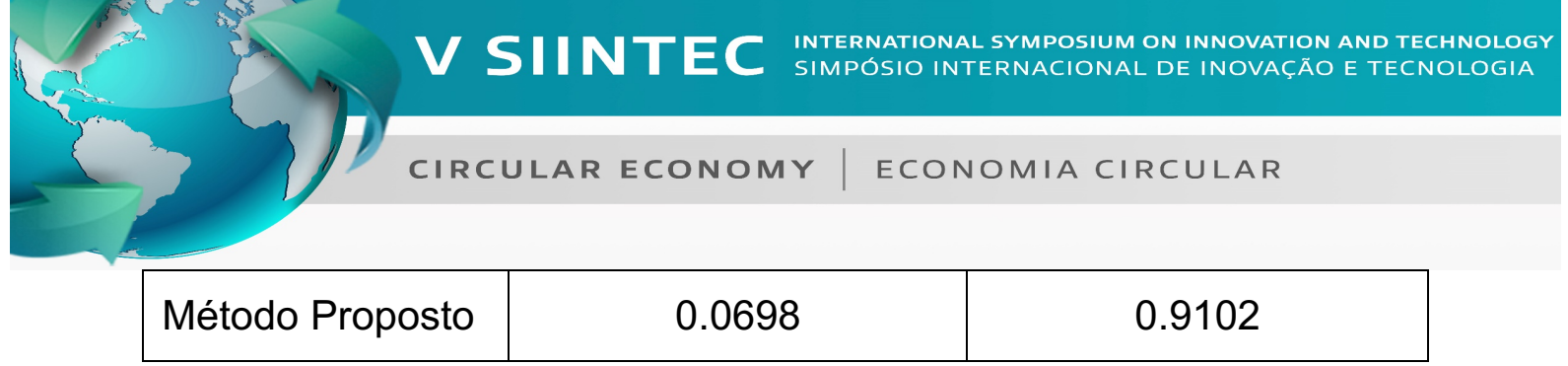

\section{CONCLUSÕES}

Este artigo delineia um método de detecção e remoção de sombras baseado no espaço de cores HSV. A maioria dos trabalhos anteriores envolveu várias imagens, juntamente com uma câmera calibrada, enquanto o método proposto é uma maneira simples e eficiente de remover sombras de imagens individuais. Análises e resultados de experimentos sugerem que a estrutura de detecção e remoção de sombra proposta é mais precisa do que os métodos de [4-7]. Mas o framework proposto precisa melhorar seu tempo de computação e resposta. A ênfase deste trabalho está na implementação do modelo, para detectar e remover sombras, usando o espaço de cores HSV. Esta ênfase também é dada para melhorar a imagem livre de sombra recuperada, corrigindo a cor, algo que vimos necessita de aperfeiçoamento. A principal conquista da estrutura proposta reside na suavidade da transição entre a imagem som para sem sombra, mantendo todos os detalhes da região sombreada intactos. É evidente que a estrutura proposta efetivamente conseguiu remover sombras de várias imagens texturizadas. Durante a realização dos experimentos, diferentes pontos de vista, condições de iluminação e distâncias variadas entre objeto e câmera frequentemente ocorriam. Deixamos esses problemas para estudos futuros.

\section{REFERÊNCIAS}

${ }^{1}$ C.X. Jiang and M.O. Ward. Shadow segmentation and classification in a constrained environment. CVGIP: Image Understanding, 1994, 59(2):213 - 225.

${ }^{2}$ S. H. Khan, M. Bennamoun, F. Sohel, and R. Togneri. Automatic shadow detection and removal from a single image. IEEE Transactions on Pattern Analysis and Machine Intelligence, 38(3):431-446, 2016.

${ }^{3} \mathrm{~N}$. Su, Y. Zhang, S. Tian, Y. Yan, and X. Miao. Shadow detection and removal for occluded object information recovery in urban high-resolution panchromatic satellite images. IEEE Journal of Selected Topics in Applied Earth Observations and Remote Sensing, 9(6):2568-2582, 2016.

\footnotetext{
${ }^{4}$ Fei Kou, Weihai Chen, Changyun Wen, and Zhengguo Li. Gradient domain guided image filtering. IEEE Transactions on Image Processing, 24(11):4528-
} 
4539, 2015.

${ }^{5}$ Anat Levin, Assaf Zomet, Shmuel Peleg, and Yair Weiss. Seamless image stitching in the gradient domain. In European Conference on Computer Vision, pages 377-389. Springer, 2004.

${ }^{6}$ Patrick Pérez, Michel Gangnet, and Andrew Blake. Poisson image editing. ACM Transactions on graphics (TOG), 22(3):313-318, 2003.

${ }^{7}$ Graham D Finlayson, Steven D Hordley, Cheng Lu, and Mark S Drew. On the removal of shadows from images. IEEE transactions on pattern analysis and machine intelligence, 28 (1):59-68, 2005.

${ }^{8}$ Hazewinkel, M, Poisson equation, Encyclopedia of Mathematics, Springer Science+Business Media B.V. / Kluwer Academic Publishers, Disponível em: <https://www.encyclopediaofmath.org/index.php/Poisson_equation>. Acesso em: 18 ago. 2019.

${ }^{9}$ T. F. Y. Vicente, M. Hoai, and D. Samaras. Leave-one-out kernel optimization for shadow detection and removal. IEEE Transactions on Pattern Analysis and Machine Intelligence, 40 (3):682-695, 2018.

${ }^{10}$ M. Xu, J. Zhu, P. Lv, B. Zhou, M. F. Tappen, and R. Ji. Learning-based shadow recognition and removal from monochromatic natural images. IEEE Transactions on Image Processing, 26(12):5811-5824, 2017.

${ }^{11} \mathrm{~K}$. He, R. Zhen, J. Yan, and Y. Ge. Single-image shadow removal using 3D intensity surface modeling. IEEE Transactions on Image Processing, 26(12):60466060, 2017.

${ }^{12}$ L. Zhang, Q. Zhang, and C. Xiao. Shadow remover: Image shadow removal based on illumination recovering optimization. IEEE Transactions on Image Processing, 24(11):4623-4636, 2015.

${ }^{15}$ Otsu, Nobuyuki. A threshold selection method from gray-level histograms. IEEE Trans. Sys., Man., Cyber. 9 (1): 62-66. 1979. 